\title{
An Autoencoder-Based Solution for IQ Constellation Analysis
}

\author{
Marc Ruiz*, Javier Morales, Diogo Sequeira, and Luis Velasco \\ Universitat Politècnica de Catalunya (UPC), Barcelona, Spain (*marc.ruiz-ramirez@upc.edu)
}

\begin{abstract}
A$ method for $I Q$ constellation analysis based on Autoencoders is proposed. Exhaustive numerical results show accurate physical metric prediction and large data compression, while providing useful model explainability.
\end{abstract}

\section{Introduction}

One of the most active fields of Artificial Intelligence (AI) application in optical networks is Optical Performance Monitoring (OPM) [1]. Specially interesting are those machine learning (ML)-based algorithms that combine the characteristics of the physical system and realtime monitoring data to estimate a complex target, e.g., nonlinear interference (NLI) noise [2]. For these approaches to properly work, not only accurate ML models need to be trained, but also precise knowledge of input physical parameters is needed. Examples of physical parameters of optical connections are the end-to-end path length and the Transmitter ( $\mathrm{Tx}$ ) launch power. Although, some works assume that physical parameters remain invariable in time, considering them strongly increases the applicability of the approaches in real deployments.

Other approaches receiving large attraction are those exploring deep learning techniques to extract information from complex, dense monitoring data inputs, without knowledge of the physical characteristics. E.g., in coherent systems with advanced Digital Signal Processing (DSP), the analysis of In-Phase and Quadrature (IQ) constellation diagrams as images can be performed by means of training Convolutional Neural Networks (CNN) to estimate the Quality of Transmission (QoT) of optical signals ${ }^{[3]}$.

In this work, we propose a method for IQ constellation analysis based on Autoencoders $(\mathrm{AE})^{[4]}$. AEs are a type of Deep Neural Networks (DNN) with two network components: the encoder and the decoder. Although AEs themselves require training, the encoder transforms the input in a reduced number of latent features in an unsupervised manner, so that the decoder is able to reconstruct the original input from the latent features space. The proposed AE-based method is presented and numerically evaluated to illustrate their potential to estimate physical lightpath characteristics, which can be afterwards used for further analysis. Moreover, data compression and explainability are intrinsic aspects of AEs that are tackled as key part of the proposed method.

\section{Scenario and approach}

The considered scenario is depicted in Fig. 1, where the $T x$ at Site $A$ and the Receiver $(R x)$ at site $B$ are connected through a lightpath established on the optical network. The AEbased IQ constellation analysis module runs at the Rx site, e.g., in the node agent ${ }^{[5]}$, and processes sets of received IQ symbols with the aim of analyzing their position in the constellation. The IQ constellation in Fig. 1 shows an example of processed signal for a 16QAM signal; one of the external constellation points $(3+3 i)$, among the ones that are affected the most by NLI noise, is zoomed in. Note that the dispersion of the symbols around their central constellation points is not uniform around $I$ and $Q$ axes; this is the resultant effect of lightpath characteristics.

Our approach aims at extending monitoring and data analysis capabilities of node agents by fully exploiting the three main inherent characteristics of AEs: 1) Unsupervised feature extraction is used to summarize large IQ constellations down to their relevant few features, which are subsequently used to estimate physical path metrics (e.g., actual distance from source to destination) by means of simpler models. These metrics are afterwards processed by some analytics application running at the Software Defined Networking (SDN) controller, e.g., to detect whether the path fits with their expected physical characteristics and enforce appropriate countermeasures, if needed; 2) Input data compression provided by the latent feature space allows collecting and storing efficiently and accurately raw compressed $I Q$ constellations, which can then be used for multiple purposes, such as training other ML models; 3 ) eXplainable Artificial Intelligence (XAl) ${ }^{[6]}$ techniques can be applied to generate additional metrics that contain valuable information, which can be processed afterwards to increase the knowledge of the systems and the physical processes involved.

\section{AE-based IQ constellation analyzer}

Fig. 2 details the main processes that run in the proposed AE-based IQ constellation analyzer. Numerical labels are provided to facilitate following the main workflow, which consists of the forward and the backward analysis phases. Without loss of generality, we assume that before lightpath operation (i.e., during the commissioning testing phase), a pre-trained $\mathrm{AE}$ 


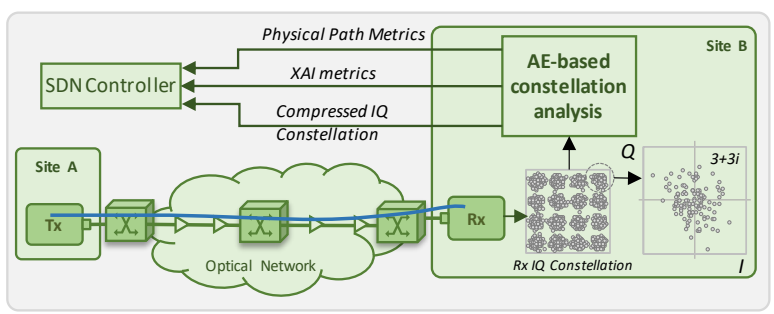

Fig. 1: Considered AE-based Analitics Architecture

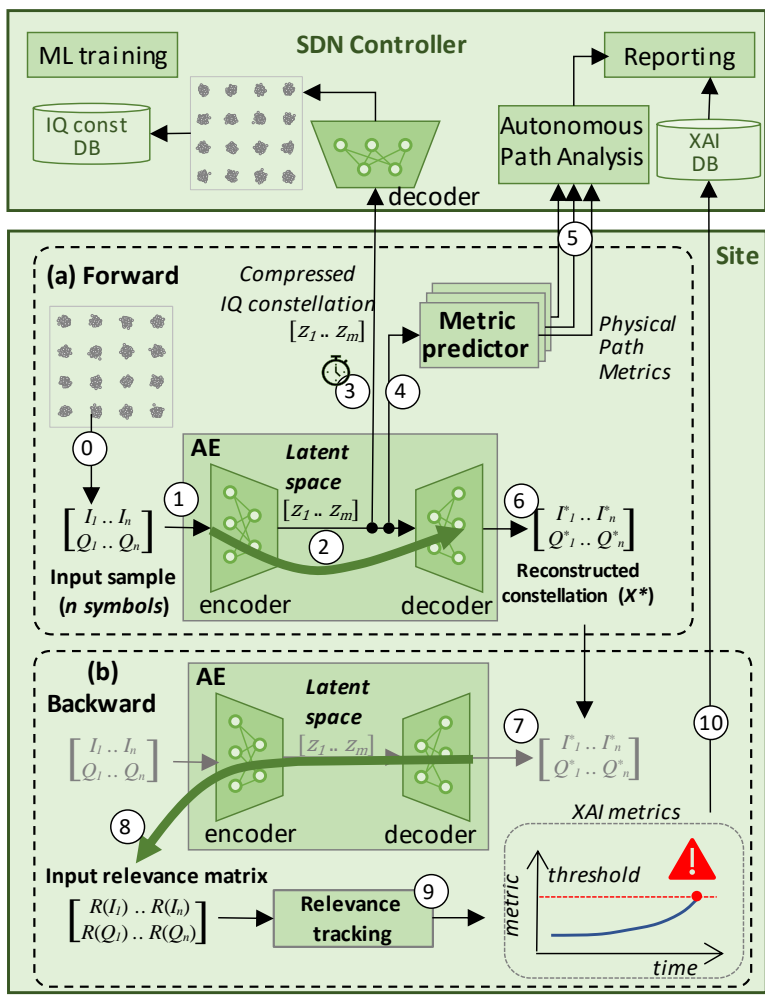

Fig. 2: AE-based analysis of received IQ constellations

model suitable for the main characteristics of the established path, e.g., length and launch power, is loaded [7]. Once in operation, an optical IQ constellation sample $X$ with $n I Q$ symbols is collected with a predefined time period (e.g., 1 min.), decoded at the Rx side, and processed (labeled 0 in Fig. 2).

After the collection of the raw IQ constellation sample, the forward analysis phase (block (a) in Fig. 2) starts by using the raw sample as input for the encoder (1). The encoder then transforms the input sample $X$ into a sample $Z$ in the latent space; $Z$ consists of $m$ values, each of them representing one feature in the latent space (2). The compressed sample $Z$ is collected periodically by the SDN controller (3). Moreover, sample $Z$ is used as input for several metric predictors (4), each producing one single metric that is sent to the controller for autonomous physical path analysis (5). Those predictors can be based on simple ML models, such as multiple linear regression models, that can be fitted to produce accurate predictions. The decoder completes the forward analysis phase by reconstructing the original sample from the latent feature space sample (6).

The backward analysis phase (block (b) in Fig. 2) is used to generate XAI metrics. Starting from the decoded sample (7), relevance backpropagation techniques [9] are applied to compute the importance of each input feature into the latent feature space. The relevance matrix $(R)$ with the same shape than that of the original sample $X$ is obtained (8). Next, relevance values are grouped (e.g., by constellation point) and its evolution in time is analyzed with the objective of finding some pattern or to compare with some predefined threshold (9). All these relevancebased data are sent as XAI metrics to the SDN controller for further reporting and analysis (10).

\section{Illustrative Results and Conclusions}

To evaluate the proposed AE-based method, a MATLAB-based simulator of a coherent WDM system has been developed to generate IQ constellations for a 16QAM@64GBd signal under different physical path characteristics. Assuming $100 \mathrm{GHz}$ channel spacing and full spectrum occupancy, signal samples containing 2,048 symbols and shaped by a root-raised cosine filter with a 0.06 roll-off-factor are generated at the $\mathrm{Tx}$ side. Then, the signal is propagated through standard single mode fiber $80-\mathrm{km}$ spans, characterized by optimal power of $-1 \mathrm{dBm}$, attenuation factor of $0.21 \mathrm{~dB} / \mathrm{km}$, dispersion parameter of $16.8 \mathrm{ps} / \mathrm{nm} / \mathrm{km}$, and nonlinear parameter of $1.141 / \mathrm{W} / \mathrm{km}$. Spans are modeled by solving the nonlinear Schrödinger equation using the well-known split-step Fourier method, whereas ideal inline optical amplification is modelled as erbium-doped fiber amplifiers with a noise figure of $4.5 \mathrm{~dB}$, introducing linear noise. Finally, at the Rx, a DSP block able to perform ideal chromatic dispersion compensation and phase recovery is considered.

With the aforementioned configuration, signals for lightpaths with total distance ranging from 80 to 2,000 km were generated. Three launch power scenarios were considered to generate signals with the same total distance but different characteristics by adding additional attenuation after the transmitter: i) optimal $(0 \mathrm{~dB})$, ii) suboptimal $(-4 \mathrm{~dB})$, and iii) degraded $(-8 \mathrm{~dB})$. Note that, as soon as attenuation increases, NLI reduces, being linear noise predominant. Thus, a total number of 3,000 signal samples were generated for training and validation purposes. For the sake of simplicity, hereafter we focus our analysis on the total path length under such different launch power scenarios.

Aiming at firstly analyzing the capability of AEs for reconstructing input IQ constellations with high fidelity, Fig. 3a shows reconstruction error as a function of the number of latent space features $(m)$. An AE with symmetric encoder and 

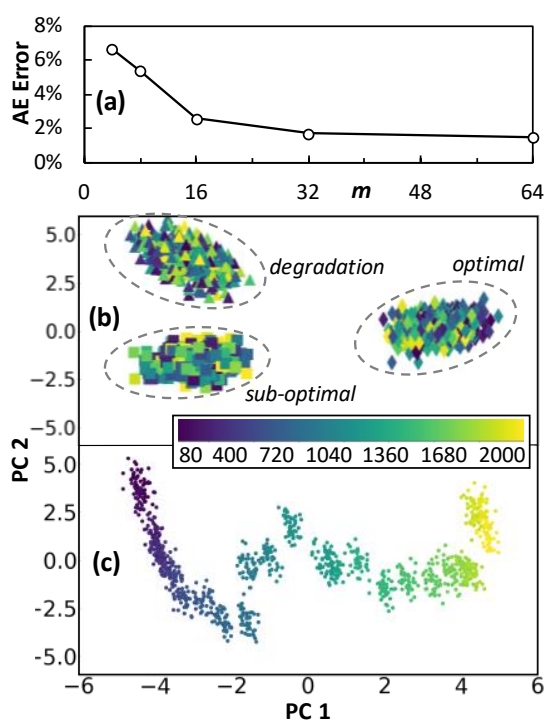

Fig. 3: AE model selection

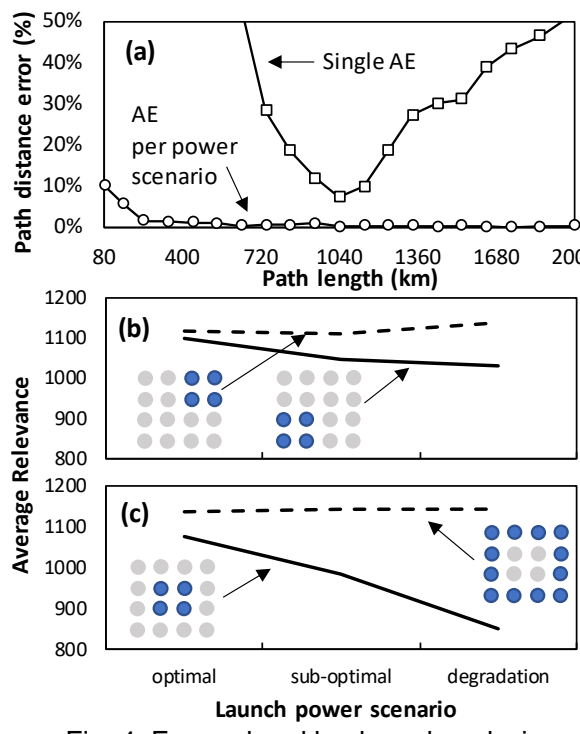

Fig. 4: Forward and backward analysis

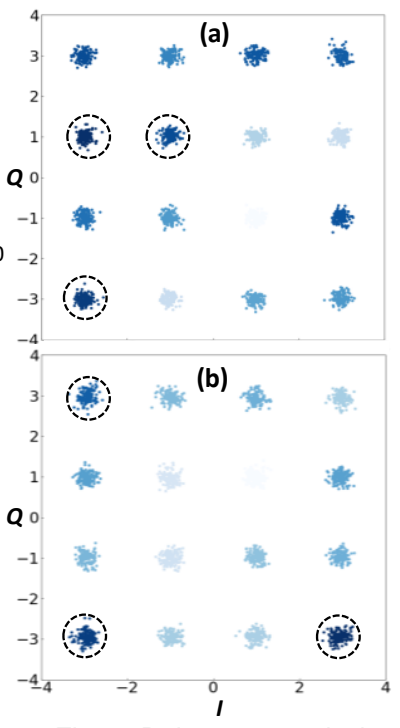

Fig. 5: Relevance analysis decoder, each with 4 hidden layers (1024, 256, 128 , and 64 ReLU neurons) was trained with data from all launch power configurations. Supported by the results, we conclude that 32 latent features are enough to reach a negligible reconstruction error lower than $2 \%$. Note that this allows achieving a compression rate of $99.2 \%$ (from 2048 complex inputs to 32 real latent space features), which validates the utilization of AEs for high compression of IQ constellations.

Now, we analyze the potential capacity of the trained $A E$ to extract relevant information for path length estimation. To this aim, we plot each sample in the latent feature space using a different color for the path length (see inset color bar) and a different marker for the launch power. To simplify visualization, we projected the original 32-dimensional feature space into just two dimensions by means of applying Principal Component Analysis (PCA) [10]. In Fig. 3b, first and second components are depicted; note that although different launch powers are clearly distinguishable in separable clusters, each of them presents a matted distribution for the distance. In view of this, we trained one AE per each launch power configuration; Fig. 3c presents the same PCA-based plot for the samples belonging to the optimal power scenario. In contrast to Fig. 3b, different lengths are now clearly separated and sorted by the latent feature space, which increases the chance of more accurate path length prediction models.

To corroborate the abovementioned analysis, multiple linear regression models have been fitted for each of the previous approaches, i.e., one unique $\mathrm{AE}$ for all power configurations or one different $A E$ for each power configuration. Each linear model receives as inputs the latent feature space sample and outputs the prediction of the path length. Fig. 4a shows the prediction error as a function of the actual path length for the two considered approaches. As it was anticipated, one single $A E$ fails in the objective of extracting relevant data for path length prediction purposes, and it produces large errors, especially for short and long distances. On the contrary, using different AEs for power configuration allows high accurate path length prediction (error below $2 \%$ for paths larger than $200 \mathrm{~km}$ ).

Once forward analysis has been carried out, we now focus on the XAl metric performance evaluation by comparing two different ways for aggregating constellation points relevance computed during backward analysis: i) perquadrant, i.e., right-upper and left-bottom quadrant (Fig. 4b); and ii) per-depth, i.e., inner and outer constellation points (Fig. 4c). In view of the figures, we can conclude that the latter is much more informative, since the relevance of inner constellation points clearly reduces when launch power reduces. Hence, this result shows that XAI analysis can be potentially used for alternative objectives, e.g., to detect whether launch power is decreasing up to a limit that it can be considered as a degradation.

Finally, two examples of constellations for optimal (Fig. 5a) and sub-optimal (Fig. 5b) scenarios are depicted, using a color scale for relevance analysis (the darker the color the more relevant). The three more relevant constellation points for each case are highlighted; results show that relevance analysis can be used to select few constellation points for further analysis, being that selection depending on the lightpath scenario.

\section{Acknowledgements}

This work has been partially supported by the EC through the MSC REAL-NET project (G.A. 813144), by the AEI/FEDER through the TWINS project (TEC201790097-R), and by the ICREA institution. 


\section{References}

[1] W. Saif et al., "Machine Learning Techniques for Optical Performance Monitoring and Modulation Format Identification: A Survey," in IEEE Communications Surveys \& Tutorials, vol. 22, pp. 2839-2882, 2020.

[2] Q. Zhuge et al., "Application of machine learning in fiber nonlinearity modeling and monitoring for elastic optical networks," IEEE/OSA Journal of Lightwave Technology (JLT), vol. 37, pp. 3055-3063, 2019.

[3] D. Wang et al., "Intelligent constellation diagram analyzer using convolutional neural network-based deep learning," Optics Express, vol. 25, pp. 1715017166, 2017.

[4] Q. Meng et al., "Relational autoencoder for feature extraction," in Proc. International Joint Conference on Neural Networks (IJCNN), 2017.

[5] L. Velasco et al., "Monitoring and Data Analytics for Optical Networking: Benefits, Architectures, and Use Cases," IEEE Network Magazine, vol. 33, pp. 100-108, 2019.

[6] F. Došilović, M. Brčić and N. Hlupić, "Explainable artificial intelligence: A survey," in Proc. International Convention on Information and Communication Technology, Electronics and Microelectronics, 2018.

[7] L. Velasco et al., "A Learning Life-Cycle to Speed-up Autonomic Optical Transmission and Networking Adoption," IEEE/OSA Journal of Optical Communications and Networking, vol. 11, pp. 226237, 2019.

[8] G. Agrawal, Nonlinear fiber optics, Academic Press, fifth edition, 2013.

[9] A. Binder et al., "Layer-wise Relevance Propagation for Deep Neural Network Architectures," in Proc. Information Science and Applications, pp. 913-922, 2016.

[10] E. Barshan et al., "Supervised principal component analysis: Visualization, classification and regression on subspaces and submanifolds," Elsevier Pattern Recognition, vol. 44, pp. 1357-1371, 2011. 